\title{
Diarrea aguda del lactante. Estudio clínico bacteriológico comparativo en grupos tratados con Bactrim, Furoxona y sin medicamento antiinfeccioso intestinal
}

DRES.: OSCAR ERRAZLIRIZ *, JOSE GILARDINO ** y MARIO PINO **.

Introducción. En el tratamiento de la diarrea aguda del lactante se han usado gran cantidad de agentes antiinfeciosos con resultados muy variables $(1,2,3,10,11,16,23,24)$. Ninguno ha logrado eficacia total y duradera, lo que se explica porque en la diarrea aguda del lactante inciden una serie de factores dependientes de los posibles agentes causales del lactante mismo que varían con cada brote de la afección y hacen dificil la valoración y la comparación de los resultados con los medicamentos empleados. Además se ha señalado que un porcentaje importante de Jas diarreas son causadas por virus $(26,27)$, agentes sobre los cuales los actuales antibióticos y quimioterápicos no tienen acción.

En el Hospital M. Arriarán se ha usado Bactrim $^{2}$ en el tratamiento de la fiebre tifoidea y en la infección urinaria $(29,30)$. Los resultados señalan que este medicamento es de gran utilidad en dichas afecciones. Esto nos indujo a pensar que también podría ser eficaz en el tratamiento de la diarrea aguda del lactante cuando ésta era provocada por gérmenes patógenos intestinales.

Nos propusimos efectuar un estudio clínico y bacteriológico usando Bactrim y comparando su acción con Furazolidona (Furoxona ${ }^{2 *}$ ), quimioterápico de gran uso actualmente en la diarrea agıda. Nos pareció importante, además, comparar estos resultados con los obtenidos en otro grupo de lactantes con el mísmo proceso patológico y al cual no se hubiese administrado ningún medicamento antiinfecioso intestinal.

\footnotetext{
* Servicio de Pediatria. Hospital M. Arriarán. Santiago, Chile. Departamento Materno-Infantil. Facultad de Mcdicina Sur. Universidad de Chile.

** Departamevto de Medicina Experimental. Facultad cle Medicina Sur Universidad de Chilé.

- TRIMETROPIN Y SULFAMEIOXASOLE. Laborator io Roche. En Cbile: Bactrimel.

i* Furazolidona. Laboratorio Eaton.
}

MATERIAL Y MÉTODO. Nuestro material lo constituyen 120 lactantes hospitalizados en la Unidad de Lactantes del Hospital M. Arriarán. Sus edades fluctuaron entre un mes y un año seis meses. Todos los enfermos fueron hospitalizados por presentar grave compromiso general, deshidratación y diarrea profusa con signos de infección enteral. Se excluyeron de este estudio pacientes que presentaban infección paraenteral (otitis, neumopatía, infección urinaria, etc.), así conno lactantes con desnutrición avanzada, incluyéndose solamente eutróficos o con desnutrición grado $\mathbf{I}$.

De los 120 casos y al azar, 50 recibieron Bactrim por vía oral, en comprimidos de $120 \mathrm{mg}$. y a dosis de $40 \mathrm{mg} / \mathrm{kg} /$ día fraccionado cada 12 horas el primer día de tratamiento y $25 \mathrm{mg} / \mathrm{kg}$ / día desde el segundo al sexto día. En caso que la diarrea persistiese más allá del sexto día, el plan contemplaba cambiar a otro medicamento.

Otro grupo de 50 lactantes recibió Furoxona en dosis de $10 \mathrm{mg} / \mathrm{kg} /$ día fraccionada cada 6 horas, por vía ora] durante 6 días. Si el proceso diarreico persistía mâs allá del sexto día el plan contemplaba cambiar a Bactrim.

En 20 lactantes comparables por el cuadro clínico, los caracteres de la diarrea, el grado de deshidratación y el estado nutritivo no se administró ningún medicamento de acción antibacteriana a nivel intestinal, por lo cual puede ser considerado como grupo control. Si en este grupo la diarrea persistía más allá de los 6 días o si la cvolución era desfavorable, se administró Bactrim.

Ningún paciente recibió, previo al estudio, terapia antibiótica por más de 24 horas.

El tratamiento general hidroelectrolítico y dietético fue semejante en todos los enfermos.

En los 3 grupos se practicaron coprocultivos seriados el primero, tercero y sexto día de hos- 
pitalización, tomando la muestra con tórula rectal. La muestra tomada el día del ingreso es, por supuesto, anterior al tratamiento. Se sembró en los siguientes medios: agar Mc. Conkey, agar E. M. B., agar con verde brillante, agar desoxicolato, agar sangre y agar sal. En todos los casos en que se encontró germen patógeno intestinal o cuando se obtuvo en el primer cultivo cepas puras de gérmenes patógenos probables, se hizo antibiograma.

La tipificación de los gérmenes patógenos se hizo por métodos de cultivo y serológicos.

Para los bacteriólogos este estudio fue ciego.

En 20 de los niños tratados con Bactrim se practicaron hemogramas y examen de orina al ingreso y al finalizar el tratamiento.

En 100 niños se practicó examen parasitológico de deposiciones.

Resultados $\mathrm{x}$ comentario. Los resultados los hemos valorado desde el punto de vista clínico y bacteriológico.

Desde el punto de vista clínico se consider6 que el resultado era favorable o bueno cuando hubo rápida mejoría general o hidroelectrolítica y cuando la diarrea mejor 6 antes de los 6 días de tratamiento. El resultado se consideró desfavorable o malo cuando la mejoría general fue lenta o arrastrada y cuando la deposición alterada persistió más allá de los 6 días.

Desde el punto de vista bacteriológico se consideró que el resultado era bueno o favorable cuando el germen aislado desaparecía en la segunda muestra de deposiciones. El resultado se consideró malo o desfavorable cuando el germen persistía en la segunda y tercera muestra.

En la tabla I se consigna el resultado de los coprocultivos en los 120 lactantes. Los gérmenes se han dividido en patógenos reconocidos, patógenos probables y no patógenos, de acuerdo a la momenclatura bacteriológica para la flora intestinal. $(4,12,13,19,20,21,22,32)$.

Podemos apreciar que un $27,5 \%$ de los gérmenes aislados pertenecen a los reconocidamente patógenos. Esta cifra es similar a la obtenida por muchos autores tanto nacionales como extranjeros, pero inferior a la obtenida por Lobos y cols. en nuestro país en 1965. (14, 5, 6, 22, 25, 31). Cabe destacar que por azar el número de gérmenes patogenos en los grupos tratados con Bactrim y Furoxona fue igual. En cambio en el grupo sin droga casi la mitad de los gérmenes aislados fue patógeno.

En cuanto al grupo patógeno probable, en nuestro material se aisló en el $24,2 \%$ de los casos. Para los bacteriólogos estos gérmenes son causales de diarrea cuando se les aisla en cultivo puro en la primera muestra de deposiciones en un lactante con diarrea aguda que no ha sido sometido a tratamiento antibiótico previo. Pertenecen a este grupo Pseudomonas, Klebsiella y algunos Proteus. Se ha discutido el mecanismo por el cual estos gérmenes habituales del intestino se hacen patógenos. Se ha descrito un mecanismo enterotóxico, por el cual estas especies microbianas en algún momento pueden producir enterotoxina y ser capaces de provocar diarrea aguda. También se ha dado gran importancia a alteraciones en la interrelación huésped-mesonero como factor primordial en el mecanismo señalado: germen, virulencia, toxicidad, número de elementos que invaden, genio epidémico por un lado; mecanismos de defensa naturales y adquiridos, edad, estado nutritivo, etc. por otro $(17,18,19,22,32)$.

Sumados los gérmenes patógenos y patógenos probables tenemos que un $51,7 \%$ de nuestro material presenta agentes capaces de provocar diarrea. Hay que agregar 2 casos de amebiasis, que indudablemente presentan diarrea aguda. El resto tiene gérmenes no patógenos. Es decir, casi la mitad de nuestros pacientes no tiene un agente infeccioso demostrado con la técnica empleada capaz de provocar diarrea. Es posible que en este grupo estén los virus, que, para muchos hoy día, son la causa de la mayoria de las diarreas. $(26,27)$.

En la tabla II se consignan los gérmenes y parásitos encontrados. Entre los patógenos el grupo coli enteropatógeno es con mucho el más frecuente. Entre los patógenos probables Klebsiella

T A B L A I

GERMENES ENCONTRADOS EN 120 LACTANTES CON DIARREA AGUDA

\begin{tabular}{lcccc}
\hline Grupo & No de casos & Patógenos & $\begin{array}{c}\text { Patógenos } \\
\text { Probables }\end{array}$ & No patógenos \\
\hline Con Bactrim & 50 & 12 & 13 & 25 \\
Con Furoxiona & 50 & 12 & 11 & 57 \\
Sin Droga & 20 & 9 & $29(24,2 \%)$ & $58(48,3 \%)$ \\
\hline T o t 21 & 120 & $33(27,5 \%)$ & & 6
\end{tabular}


GERMENES PATOGENOS EN I2O LACTANTES CON DIARREA AGUDA

\begin{tabular}{lccccc}
\hline Germen & Con Bactrim & Con Furoxona & Sin Droga & Total & $\%$ \\
\hline E. Coli 0 111 B 4 & 3 & 6 & 7 & 16 & 48,6 \\
E. Coli 0 119 B 4 & 3 & 3 & 0 & 6 & 18,1 \\
Shigella Flexneri & 3 & 0 & 1 & 4 & 12,1 \\
E. Coli 0 125 B 15 & 1 & 1 & 0 & 2 & 6,1 \\
E. Coli 0 55 B 5 & 0 & 2 & 0 & 2 & 6,1 \\
E. Coli 0 127 B 8 & 0 & 0 & 1 & 1 & 3,0 \\
E. Coli 0 86 B 7 & 1 & 0 & 0 & 1 & 3,0 \\
Salmonella Tipbirnurium & 1 & 0 & 1 & 3,0
\end{tabular}

GERMENES PATOGENOS PROBABLES EN 120 LACTANTES CON DIARREA AGUDA

$\begin{array}{llllrr}\text { Klebsiella Preumoniac } & 5 & 3 & 2 & 10 & 34,4 \\ \text { E. Coli } & 2 & 4 & 1 & 7 & 24,2 \\ \text { E. Coli-Mutab. } & 3 & 3 & 0 & 6 & 20,7 \\ \text { PS. Aeruginosi } & 2 & 1 & 1 & 4 & 13,9 \\ \text { Proteus Mir. } & 1 & 0 & 1 & 2 & 6,8\end{array}$

PARASITOS EN VBJ LACTANTES CON DIARREA AGUDA

E. Histolítica 1 1

0

1

2

1,7

TA B L A I II

EVOLUCION CLINICA DE 120 LACTANTES CON DIARREA AGUDA

\begin{tabular}{lccccc}
\hline Grupo & No de casos & Favorable & $\%$ & Desfavorable & $\%$ \\
\hline Bactrim & 50 & 43 & 86 & 7 & 14 \\
Furoxona & 50 & 57 & 74 & 13 & 26 \\
Sin droga & 20 & 8 & 40 & 12 & 60 \\
\hline
\end{tabular}

pneumoniae. Dentro del grupo coli enteropatógenos el serotipo $0111 \mathrm{~B} 4$ fue el que se aisló con mayor frecuencia. $(48,6 \%)$.

Nuestros hallazgos confirman que en la actualidad en nuestro país el grupo coli enteropatógeno es prevalente en la etiología de la diarrea aguda del lactante. En relación a los géneros Shigella y Salmonella se aprecia un aumento del primero. Esto puede deberse a un deterioro de la higiene ambiental de la población atendida por nuestro Hospital, ya que se sabe que mantención de la Shigella está en relación directa con Ia baja higiene ambiental. $(7,8,9,10,11,15,16,22$, 28).

Entre los parásitos se encontraron dos casos de Entameba histolítica.

En la tabla III anotamos la evolución clínica. Se puede apreciar que el porcentaje de me- joría clínica es francamente superior en los grupos tratados con drogas, y que en estos hay cierta superioridad de Bactrim sobre Furoxona (86 y $74 \%$ respectivamente). Uno de los 7 casos de evolución desfavorable con Bactrim tenía amebiasis que mejoró con Emetina. La evolución es favorable sólo en el $40 \%$ de los casos que no recibieron tratamiento antiinfeccioso intestinal. Este porcentaje no es despreciable y nos revela que otros factores, además del germen, influyen en la evolución clínica del lactante con diarrea aguda. Un paciente del grupo sin droga que presentó evolución desfavorable tenía amebjasis que me. joró con emetina.

Un paciente que presentó evolución clínica desfavorable en el grupo tratado con Furoxona y 10 del grupo sin droga también con evolución desfavorable recibieron posteriormente Bactrim. 
TA B A I V

EVOLUCION CLINICA DE U LACTANTES TRATADOS CON BACTRM LUEGO DE FRACASAR CON FUROXONA O SIN DROGA

\begin{tabular}{ccccc}
\hline No de casos & Favorable & $\%$ & Desfavarabic & $\%$ \\
\hline 11 & 11 & 100 & 0 & 0
\end{tabular}

EVOLUCION CLINICA DEL TOTAL DE CASOS TRATADOS CON BACTRIM

61

54

88,5

7

11,5

T A B L A V

EVOLUCION BACTERIOLOGICA DE I2O LACTANTES CON DIARREA AGUDA

A. CON GERMENES PATOGENOS

\begin{tabular}{lccccr}
\hline Grupo & No de casos & Favorable & $\%$ & Desfavarable & $\%$ \\
\hline Bactrim & 12 & 8 & 66,6 & 4 & 33,3 \\
Furoxona & 12 & 8 & 66,6 & 4 & 33,3 \\
Sin droga & 9 & 2 & 22,2 & 7 & 77,7 \\
\hline
\end{tabular}

B. CON GERMENES PATOGENOS PROBABLES

\begin{tabular}{lrrrrr}
\hline Bactrim & 13 & 10 & 76,9 & 3 & 25,1 \\
Furoxona & 11 & 7 & 63,6 & 4 & 56,4 \\
Sin droga & 5 & 2 & 40,0 & 3 & 60,0 \\
\hline
\end{tabular}

T A B L A VI

SENSIBILIDAD A BACTRIM Y FUROXONA DE GERMENES PATOGENOS Y PATOGENOS PROBABLES

\begin{tabular}{|c|c|c|c|c|c|}
\hline \multirow[b]{2}{*}{ Germen } & \multirow[b]{2}{*}{ No de casos } & \multicolumn{2}{|c|}{ Bactrim } & \multicolumn{2}{|c|}{ Furoxona } \\
\hline & & Sens. & Resist. & Sens. & Resist. \\
\hline E. Coli 0111 B 4 & 16 & 15 & 1 & 10 & 6 \\
\hline E. Coli 0119 B 14 & 6 & 4 & 2 & 5 & 1 \\
\hline Shigella Flexneri & 4 & 1 & 3 & 4 & 0 \\
\hline E. Coli 0125 B is & 2 & 2 & 0 & 1 & 1 \\
\hline E. Coli 0 55 B 5 & 2 & 2 & 0 & 2 & 0 \\
\hline E. Coli 0 127 B 8 & 1 & 1 & 0 & 0 & 1 \\
\hline E. Coli 036 B 7 & 1 & 1 & 0 & $i$ & 0 \\
\hline \multicolumn{6}{|l|}{ Salmonella } \\
\hline Tiphimutium & 1 & 1 & 0 & 0 & 1 \\
\hline Klebsiella Pneumoniae & 10 & 3 & 7 & 10 & 0 \\
\hline E. Coli & 7 & 6 & 1 & 6 & 1 \\
\hline E. Coli-Mut. & 6 & 6 & o & 6 & 0 \\
\hline Pseudomona Aeruginosi & 4 & 0 & 4 & $\mathbf{0}$ & 4 \\
\hline Proteus Mir. & 2 & 2 & 0 & 2 & 0 \\
\hline
\end{tabular}


Todos tuvieron evolución favorable. Esto hace que el total de lactantes en los que se usó esta droga sea de 61 y la evolución fue favorable en el $88,5 \%$ de ellos. (tabla IV).

En la tabla $\mathrm{V}$ se puede apreciar la evolución bacteriológica. Los dos tercios de los enfermos $(66,6 \%)$ tratados con droga (Bactrim o Furoxona) mejoran bacteriológicamentc. No hay diferencia entre Bactrim y Furoxona. En el grupo sin droga hay mejoría sólo en 4 de los 14 pacientes $(28,5 \%)$ con germen patógeno o patógeno probable.

Analizando en conjunto las tablas III, IV y $V$ podemos apreciar que tanto Bactrim como Furoxona son útiles en el tratamiento de la diarrea aguda del lactante cuando ésta es provocada por gérmenes patógenos o patógenos probables. El porcentaje de mejoría clínica de $88,5 \%$ obtenido con Bactrim es bueno. Podemos ver también que el no dar medicamento antiinfeccioso intestinal cuando la diarrea aguda del lactante es provocada por los gérmenes antedichos ticne riesgo, ya que el porcentaje de mcjoría clínica es bajo y sobre todo el porcentaje de mejoría bacteriológica on los pacientes con germen patógeno es muy bajo. $(22,2 \%)$.

Podemos ver también en todos los grupos que e] porcentaje de mejoría clínica es superior al de la mejoría bacteriológica. Este hecho, junto al ya comentado de mejoría de pacientes sin droga con germen patógeno revela que hay, además, del germen causal, otros factores que inciden en los resultados.

En la tabla VI hemos colocado la sensibilidad de los gérmenes a Bactrim y Furoxona. Podemos apreciar que, en el grupo patógeno, el grupo coli enteropatógeno es muy sensible a Bactrim. En cambio Shigella es resistente. Entre los patógenos probables Klebsiella es resistente, E. Coli mutabilis bastante sensibles y Pseudomonas resistente. No se puede decir nada aún sobre Salmonełla porque sólo se aisló en un paciente. En cuanto a Furoxona, los coli enteropatógenos son más resistentes que a Bactrim. Shigella es más sensible y entre los patógenos probables, Klebsiella aparece como muy sensible.

En cuanto a tolerancia podcmos decir que Bactrim fue bien tolerado en todos los pacientes. Tampoco se demostraron alteraciones en el hemograma y sedimento urinario en ninguno de los 20 enfermos a los cuales se les practicaron dichos análisis.

\section{RESLMEN Y CONCLUSIONES}

En un grupo de 120 lactantes con diarrea aguda $y$ deshidratación se valora clínica y bacteriologicamente el efecto de la terapia antibacteriana intestinal. Se excluyeron del estudio los enfermos con infecciones parenterales y los desnutridos de $2^{\circ}$ y 3 er. grado.

Al azar, 50 pacientes recibieron Bactrim a dosis de $40 \mathrm{mg} / \mathrm{kg} /$ da el primer día para luego continuar con $25 \mathrm{mg} / \mathrm{kg} /$ dia hasta completar 6 dias. Otros 50 enfermos recibieron Furoxona a dosis de $10 \mathrm{mg} / \mathrm{kg} /$ dia durante 6 dias. Los 20 enfermos restantes no recibieron terapia antiinfecciosa. El tratamiento general, hidroelectrolitico y dietético fue iguat en los tres grupos.

En el $27,5 \%$ de los casos se aislaron gérmenes patógenos, siendo los del grupo coli enteropatógeno los más frecuentes. En el $24,2 \%$ se aislaron gérmenes patógenos probubles. Se analizan y comparan estos resultados. En dos casos se demostró la presencia de Entameba histolitica.

Se obtuvo evolución clinica favorable en un atto porcentaje de los casos tratados con Bactrim y Furoxona con leve superioridad para Bactrim (86 y $74 \%$ respectivamente). En los pacientes que no recibieron droga la evolución clínica fue favorable en sólo el $40 \%$ de los casos, porcentuje que de todas maneras nos indica que otros factores, además del germen, están influyendo en la evolución clinica del lactunte con diarréa aguda.

La mejoría bacteriológica, algo más baja que la mejoria clínica, se obtiene tambien en un alto porcentaje de los casos tratados con Bactrim $(66,6$ y 76,9\% con gérmenes patógenos y patógenos probables respectivamente). Con Furoxona también los resultados fueron satisfactorios $166,6 \%$ con gérmenes patógenos y $63,6 \%$ con patógenos probables). En el grupo tratado sin droga la evolucion favorable fue muy inferior $(22,2 \%$ con germen patógeno $y 40 \%$ con patógeno probable).

Bactrim se revela en este estudio como un medicamento muy bien tolerado y de alta eficacia en la diarrea aguda del lactante, especiamente cuando el agente caisal pertenece al grupo coli enteropatógeno.

\section{SUMMARY AND CONCLUSIONS}

Intestinal antibacterial therapy is clinically and bacteriologycaly evaluated in 100 infants affected with acute diarrhea and dehydration. Infants with paraenteral infection or severe malnutrition were not included. 50 patients were treated with Bactrim. The dose was 40 $\mathrm{mg} / \mathrm{kg} /$ day first day and $25 \mathrm{mg} / \mathrm{kg} / \mathrm{day}$ from the 2 nd. to the 6th. day. 50 patients were treated with Furoxona. The dose was $10 \mathrm{mg} / \mathrm{kg} /$ day from the ist. to the 6 th day. 20 patients did'nt receive any antibacterial drug.

General, hydroelectrolytic and dietary therapy was the same to all palients.

Intestinal pathogens were isolaicd in $27,5 \%$. E. coli enteropathogenic group was the most frecuently 
isolated. Probably intestinal pathogens were isolated in $24,2 \%$. These results are analyzed and compared. E. Hyswolitica was isolated in 2 patients.

Oood clinical results were obtained in patients treated with Bactrim and Furoxona. (80 and $74 \%$ respectively). Clinical results were poor in patients without treatment. $(40 \%)$. Good bacteriological results were obtained in patients treated with both drugs, but inferior than clinical results. Poor results were obtained in patients without antibacterial treatment. $(22,2 \%$ in patients with intestinal pathogens).

Bactrim is a new drug very effective in treatment of acute diarthea in infants. Tolerance was good. No side effect was demostrated.

\section{REFERENCIAS}

1.-Aguilo, C., Rizzardini, M., Manubens, F., Schusrer, $A$. Tratamiento de la diarrea aguda con Quixalin. Pediatría, 8; 314, 1965.

2.-Aguilo, C. Rizzardini, M. Manubens, F. Schuster, $A$. Tratamiento de la diarrea aguda grave con Gentamicina. Pediatría. 9; 74, 1966.

3.-Aguiló, C., Soriano, H., Rizzardini, M., Macaya J. Ampicilina en el tratamiento de Ja diarrea aguda del lactante. Pediatría, 11, 263, 1968.

4.- Carpenter, $P$. International Bulletin of Bacteriological nomenclature and Taxonomy .13; No 2; 69, 1963.

5.-Cid, L., González, $M$. Diarreas infecciosas del lactante. Rev. Chilena de Pediatría. 16; 573, 1945.

6.-Costa, A. Arcaya, O. Etiología de las diarreas infantiles. Rev. Chilena de Ped. 27; 233, 1956.

7.-Costa, A. y Arcaya, O. Escherichia coli patógeno en la diarrea del niño. Rev. Chilena de Ped. 26: 451, 1955.

8.- Costa, A, y Arcaya, O. Los colibacilos enteropatogenos. Rev. Chilena de Ped. 26: 476, 1955.

9.-Cosia, $A$. Disentería bacilar en la infancia. Rev. Chilena de Ped. 16: 646, 1945.

10,-Donoso, P., Montoya, C., Contreras, J. Diarrea aguda por Shigella en el lactante. Rev. Chilena de Ped. 35: 1151, 1964.

11.-Donoso, P., Ackel, A., Montoya, C., Herrera, J., Gilabert, B., Martinez, I., Sanz, A. Sulfato de Gentamicina y Neomicina en las diarreas agudas del lactante. Rev. Chilena de Ped. 37: 589, 1966.
12.-Ewing, W. H. y Edwards, P. R. International Bulletin of Bacteriological Nomenclature and $\mathrm{Ta}$ xonomy. 13: Ne 2: 95, 1963.

13.- Ewing. W. H. International Bulletin of Bacteriological Nomenclature and Taxonomy. 10: No 1, 1960.

14.-Garcés, C. y Parada, E. Etiología de las diarreas del lactante. Rev. Chilena de Ped. 16: 695, 1945.

15.-Garcés, C. Infecciones entéricas del lactante. Rev. Chilena de Ped. 16: 625, 1945.

16.-Garcés, H., Muñoz, R., Guzmán, A., Arcaya, $O$. Estuđio clínico y terapéutico de la Shigellosis del laclante. Rev. Chilena de Ped. 37: 597, 1966.

17.-Gordon, J. E., Behar, N., Scrimshaw, N. Acute diarreal disease in less developed countries. Gastrointestinal disease. Technical discussions. XIV Meeting of the FAO. Directing Conncil. OMS. 100: 6. Dec. 1964.

18.-Gordon, J. E. Control de las infecciones entéricas. Organización Panamericana de la Salud. No 100: 6. Jun. 1964.

19.-- Jordan and Burrows. Bacteriology. 13 ${ }^{\text {a }}$ edición. W. B. Saunders, Philadelphia and London, 1943.

20.- Kaufman, F. Enterobactriaceae. Ejnar Monkgaard Publisher. Copenhagen. 1951.

21.- Le Minor, $L$. Enterobactéries. $2^{a}$ editión. Editions de la Tourelle. Paris, 1962.

22.-Lobos, H., Aguiló, C., Romero, H. Bacteriología de las diarreas agudas del lactante hospitalizado. Pediatría. 8: 280, 1965.

23.-Manterola, A., Undurraga, O. Meneghello, J. Estreptomicina en el tratamiento de las diarreas del lactante y del prematuro en el medio hospitalario. Rev. Chilena de Ped. 22: 1, 1951.

24.-Meneghello, J., Undurraga, O., Manterola, A., Sepúlvedo, H. Estreptomicina en el tratamiento de las diarreas infantiles. Rev. Chilena de Ped. 20: $1,1949$.

25.-Prado, E., Dussert, E., González, C. Bacteriologia de las diarreas infantiles. Bol. Inst. Bacteriológico de Chile. XI; 32. 1959.

26.- Ramos Alvarez, $M$. Virus y Bacterias en la diarrea de niños. Bol. Hosp. Infantil de México. XIX: $143,1962$. 
27.-Ramos Alvarez, M, Sabin, A. Enteropatogenic viruses and bacterias in diarrbea, J. A. M. A. 167: No 2. May $10,1958$.

28.--Rodríguez, $M$. Rizzardini, $M$. Investigación de las cepas 0111,055 y 026 de la Escherichia coli enteropatógena en 41 casos de diarrea en prematuro. Pediatría, 1: 141, 1958.

29.-Sempresto, L., Gamboa, R., Siva, M., Gaitua, $M$. $T$. Tratamiento con Bactrion de la fjebre tifoídea y paratifoídea en el niño. Rev. Chilena de Ped. 40: 515, 1969.

30.- Silva, M., Valdés, B., Bravo, I., Silva, M., Saitua, M. T. Ensayo terapéutico en la infección urinaria del niño con Bactrim. Rev. Chilena de Ped. 42: 469, 1971.

31.-Symon, J. Bauza, J. Diarreas agudas no específicas del lactante. Rev. Chilena de Ped. 16: 555, 1945.

32.-Topley and Wilson. Bacteriology. 4\% Ed. Edward Arnold Ltda. London, 1957. 